\title{
Diameter-selective separation of double-walled carbon nanotubes
}

\author{
Jin Hee Kim, ${ }^{1}$ Masakazu Kataoka, ${ }^{1}$ Yoong Ahm Kim, ${ }^{1, a)}$ Daisuke Shimamoto, ${ }^{1}$ \\ Hiroyuki Muramatsu, ${ }^{1}$ Takuya Hayashi, ${ }^{1}$ Morinobu Endo, ${ }^{1}$ Mauricio Terrones, ${ }^{2}$ and \\ Mildred S. Dresselhaus ${ }^{3}$ \\ ${ }^{1}$ Faculty of Engineering, Shinshu University, 4-17-1 Wakasato, Nagano-shi 380-8553, Japan \\ ${ }^{2}$ Department of Advanced Materials, IPICYT, San Luis Potosi 78210, Mexico \\ ${ }^{3}$ Massachusetts Institute of Technology, Cambridge, Massachusetts 02139-4307, USA
}

(Received 4 September 2008; accepted 5 November 2008; published online 3 December 2008)

\begin{abstract}
Here, we report a simple and effective way of separating double-walled carbon nanotubes as a function of their diameter using individually dispersed nanotube solutions with the aid of long and random single-stranded DNA. The subtle $p \mathrm{H}$ change in nanotube solutions gives rise to the preferential coagulation of large-diameter tubes and allows the easy preparation of small-diameter tubes. The stronger van der Waals forces between large-diameter tubes, combined with the decreased solubility of DNA in water at low $p \mathrm{H}$, lead to the preferential agglomeration of large-diameter tubes. (C) 2008 American Institute of Physics. [DOI: 10.1063/1.3039790]
\end{abstract}

Carbon nanotubes have attracted the attention of scientists as they are considered to be ideal macromolecules exhibiting unique physical and chemical properties that are useful for various potential applications. Since all known synthetic routes for producing nanotubes yield samples containing mixtures of carbon nanotubes with different diameters and chiralities, extensive efforts have been devoted to finding an effective way of producing or isolating particular types of carbon nanotubes. It has been reported that singlewalled carbon nanotubes (SWNTs) can be effectively sorted using surfactants in conjunction with multiple density gradient ultracentrifuges ${ }^{1}$ or by using aromatic polymers. ${ }^{2}$ Very recently, LeMieux et al. ${ }^{3}$ reported the efficient fabrication of self-sorted and self-assembled SWNT networks for thin-film transistors through the selective deposition of metallic and semiconducting tubes on the controlled surface of a wafer. DNA has also been used as an effective dispersion and separation agent for SWNTs in water on the basis of its ability to wrap itself around the sidewall of carbon nanotubes due to van der Waals attraction between the DNA molecule and the carbon nanotubes. ${ }^{4,5}$ Thus, both theoretical and experimental studies have been carried out in order to understand the nature of the interaction between DNA and nanotubes. ${ }^{6-9}$

On the other hand, double-walled carbon nanotubes (DWNTs) have attracted considerable attention since their intrinsic coaxial structure gives rise to intriguing electronic and optical properties. ${ }^{10}$ In addition, these double-walled tubes possess clear advantages over SWNTs for various specific applications such as superior mechanical properties and structural and thermal stability. ${ }^{11}$ In the present paper, we present the development, with the aid of optical spectroscopy, of an efficient method for the separation of DWNTs (as a function of their diameter) by dispersing the DWNTs in solutions of long and random single-stranded DNA (ssDNA) sequences. The subtle $p \mathrm{H}$ change in the DNA/DWNT solutions gives rise to the preferential coagulation of largediameter tubes and allows the easy precipitation of the smalldiameter DWNTs. In addition, flocculated large-diameter tubes can be separated at low $p \mathrm{H}$ by using a mild centrifuge

\footnotetext{
a) Author to whom correspondence should be addressed. Electronic mail: yak@endomoribu.shinshu-u.ac.jp. Tel.: +81-26-269-5212. FAX: +81-26269-5208.
}

and then easily redispersed in a stock DNA solution with a simple vortex mixer. We have found that this separation technique is effective for DWNTs dispersed in long and random DNA solutions.

We used highly pure ( $99 \%)$, highly crystalline DWNTs in which nanotubes with an outer diameter of $\sim 1.6 \mathrm{~nm}$ were packed in hexagonal arrays within the bundles. $^{10}$ The purity of these DWNTs is based on the absence of the $D$-band in the Raman spectra and other sample characterization. ${ }^{10}$ Subsequently, the prepared highly pure DWNTs $(\sim 4 \mathrm{mg})$ were individually dispersed (or isolated) in an aqueous solution $(10 \mathrm{ml})$ with the help of ssDNA and the surfactant sodium dodecyl-benzene sulfonate (SDBS) under strong sonication (KUBOTA UP50H, $\sim 470 \mathrm{~W} / \mathrm{cm}^{2}$ ) for $1 \mathrm{~h}$ at $4{ }^{\circ} \mathrm{C}$ and subsequent ultracentrifugation (Optima Max-XP, Beckman Coulter, 240000 g). Finally, we measured the UV-visible absorption spectra (SolidSpec-3700, Shimazu) and the photoluminescence (PL) maps (NIR-PL system, Shimazu) of the DNA/DWNT solutions that were prepared in this way.

As shown in Fig. 1, DWNTs dispersed in a solution containing long and random ssDNA sequences exhibited well-resolved and sharp optical absorption peaks due to

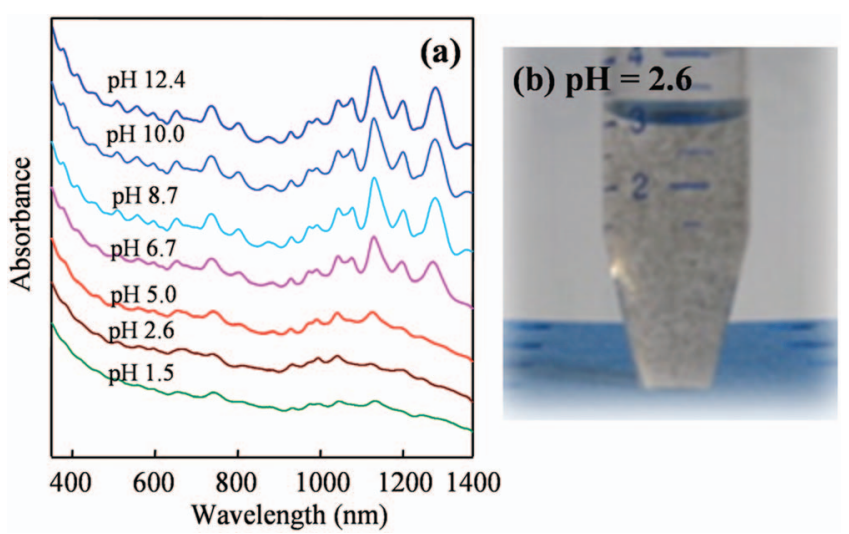

FIG. 1. (Color) (a) UV-visible absorption spectra of DWNTs dispersed in a solution of long and random ssDNA for various values of $p \mathrm{H}$. (b) The DNA/DWNT solution at $p \mathrm{H} 2.6$ contained clearly visible agglomerates. Note that individually dispersed DWNTs interact with each other at basic and neutral $p \mathrm{H}$ values and aggregate into wormlike agglomerates at acidic $p \mathrm{H}$. 

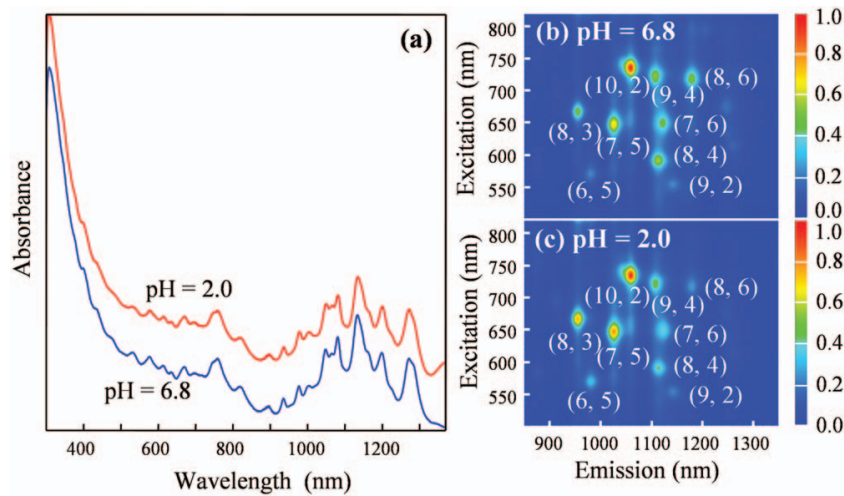

FIG. 2. (Color) (a) UV-visible absorption spectra of SDBS-dispersed DWNT solutions at $p \mathrm{H} \quad 6.8$ and 2.0 and [(b) and (c)] their corresponding PL maps. The color represents the PL intensity on a linear scale. The values of $n$ and $m$ indicate the chirality of the nanotube (Refs. 12-14).

their excitonic transitions between van Hove singularities [Fig. 1(a)], thus indicating that nanotubes were isolated. When considering the diameter distribution of our DWNTs (inner diameter $=0.6-0.9 \mathrm{~nm}$ and outer diameter $=1.4-2.0 \mathrm{~nm}$ ), the intensified peaks at $900-1300 \mathrm{~nm}$ can be assigned to $E_{22}^{S}$ excitonic transitions of the outer tubes and to $E_{11}^{S}$ transitions of the inner tubes, and the absorption peaks located at $500-800 \mathrm{~nm}$ can be assigned to the $E_{22}^{S}$ transitions of the inner tubes if we assume the absence of SWNTs in our DWNT sample. When the solution $p \mathrm{H}$ was decreased to below 6.7, the strong optical absorption peaks at 900-1300 nm became weak and broad. The sidewall protonation of the nanotubes is known to be the main reason for the appearance of broad absorption peaks in sodium dodecyl sulfonate/ SWNT solutions for $p \mathrm{H}<3$. $^{12}$

In contrast, SDBS-dispersed DWNT solutions displayed drastically different behaviors at low $p \mathrm{H}$. Even though the $p \mathrm{H}$ was decreased to 2.0 , no distinctive changes were found in the optical absorption spectra and the PL maps (Fig. 2), indicating that the inner tubes of the DWNT were completely protected by the outer tubes. This protective function of the outer tubes is very helpful in drawing conclusions about the assignment of the optical absorption peaks to specific optical transitions. The absorption peaks between 900 and $1300 \mathrm{~nm}$, assigned to the $E_{11}^{S}$ transitions of SWNTs and the $E_{22}^{S}$ transitions of the outer tubes, should be quenched (or should disappear) through the direct protonation of the sidewall. However, the absence of changes in the optical absorption spectra of the SDBS/DWNT solutions at different $p \mathrm{H}$ values provides experimental evidence that the observed absorption peaks in the $300-1350 \mathrm{~nm}$ region originate exclusively from the inner tubes of the DWNTs, from where it can be concluded that the inner tubes of the DWNT have higher extinction coefficient than SWNTs. However, the protonation effect is not sufficient for explaining the broadened and weakened absorption peaks at $900-1300 \mathrm{~nm}$ in the DNA/ DWNT solutions for $p \mathrm{H}<6$ since the protonation only occurs on the outer tubes of the DWNTs. We observed noticeable black wormlike agglomerates when the $p \mathrm{H}$ was decreased to 2.6 [Fig. 1(b)]. The bundled or nonisolated SWNTs in the solution were known to exhibit broad and weak absorption spectra due to intertube interaction, which decreased their excited state lifetimes. ${ }^{13}$

In order to confirm the aforementioned $p \mathrm{H}$-sensitive changes, we measured the PL maps of the DNA/DWNT so-

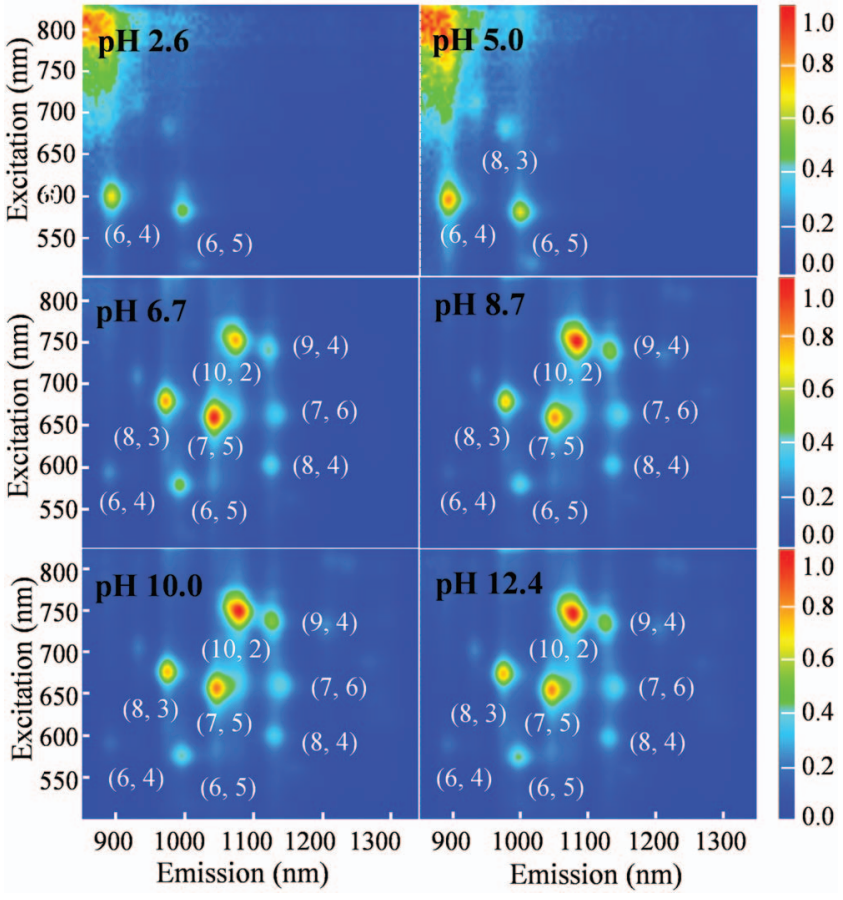

FIG. 3. (Color) PL maps of DNA-dispersed DWNT aqueous solutions prepared at different $p \mathrm{H}$ values. For $p \mathrm{H}<6.7$, only two PL species corresponding to $(6,4)$ and $(6,5)$ inner tubes survived. The color represents the PL intensity on a linear scale. The values of $n$ and $m$ indicate the chirality of the nanotube (Refs. 12-14).

lutions at different $p \mathrm{H}$ values (Fig. 3). Each peak in the PL map corresponds to an excitation involving the second excitonic transition $E_{22}^{S}$ of semiconducting inner tubes of the DWNT and emission from the first excitonic transition $E_{11}^{S}$ of semiconducting inner tubes of the DWNT. From the PL maps, we are able to get the atomic structure of the inner tubes of the DWNT [e.g., their chiral indices $(n, m)] .{ }^{13} \mathrm{We}$ observed a redshift $(\sim 45 \mathrm{meV})$ of the $E_{11}$ emission from the solution in Fig. 3 as compared to the SDBS/DWNT solution (Fig. 2), which can be explained by the changes in the dielectric constants due to the effects of the environment. ${ }^{15}$ DNA/DWNT solutions for $p \mathrm{H}$ between 6.8 and 12.4 exhibited three strong PL peaks, which can be ascribed to the inner tubes with chiralities $(8,3),(7,5)$, and $(10,2)$. However, when the $p \mathrm{H}$ was decreased to under 6.8 , only two PL species corresponding to $(6,4)$ and $(6,5)$ tubes were observed (Fig. $3)$. It is considered that the entrapped metallic nanotubes within the agglomerate might quench electronic excitations on adjacent semiconducting tubes, thus preventing their luminescence. ${ }^{13}$

We then subjected the DNA/DWNT solutions prepared at $p \mathrm{H}<6.7$ to centrifugation $(20000 \mathrm{~g})$ in order to remove or separate the agglomerates. DNA/DWNT solutions prepared at $p \mathrm{H}$ of 3.3 [Figs. 4(a) and 4(b)] exhibited broad and weak absorption peaks and very weak PL intensities, which can be attributed to the small $(6,4)$ inner tubes. The DNA/ DWNT solution remaining after the centrifugation [Figs. 4(a) and $4(\mathrm{c})$ ] contained tubes with small diameters while the agglomerate, which was dispersed in a stock DNA solution with a vortex mixer for 10 min [Figs. 4(a) and 4(d)], contained tubes with large diameters. From the centrifuged DNA/DWNT solution with a $p \mathrm{H}$ of 2.7 , we were able to obtain two PL species corresponding to $(6,4)$ and $(6,5)$ tubes [Fig. 4(e)]. However, the agglomerate precipitated from 

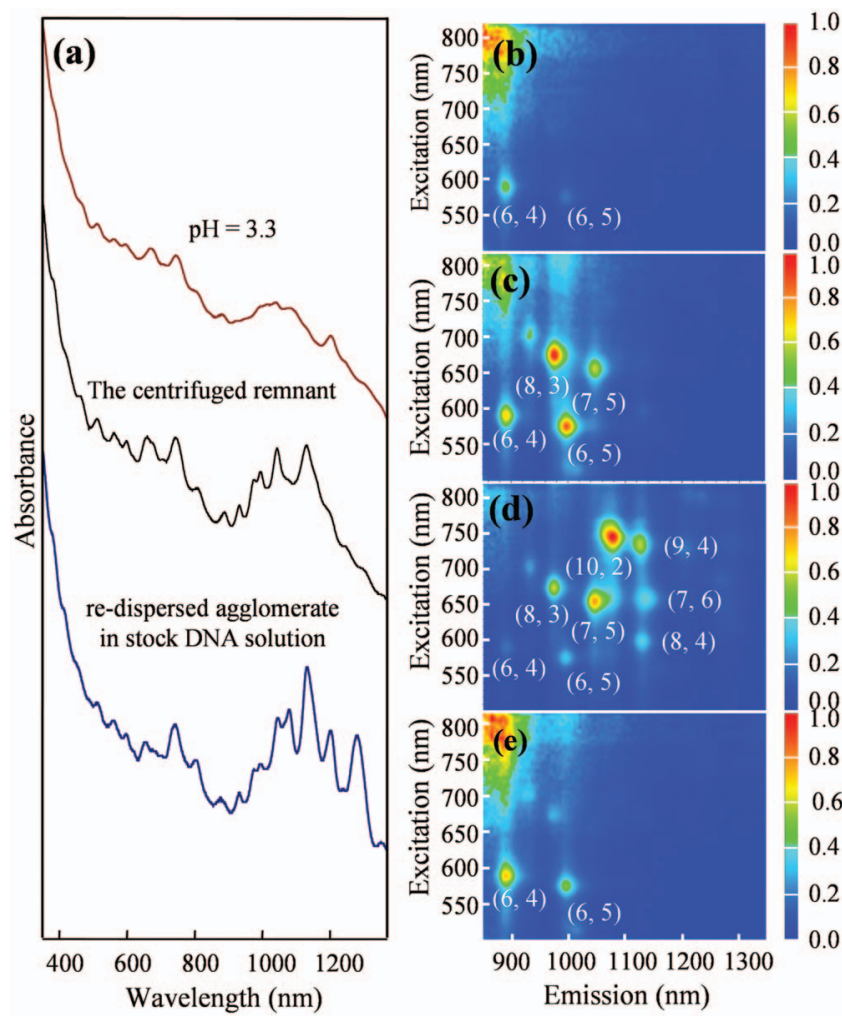

FIG. 4. (Color) (a) UV-visible absorption spectra of the DNA/DWNT solu-

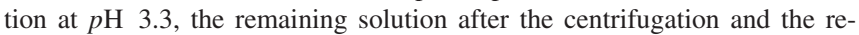
dispersed solution, and their respective PL maps [(b)-(d)]. (e) PL map of the centrifuged DNA/DWNT solution at $p \mathrm{H} 2.0$ showing the presence of the $(6,4)$ and $(6,5)$ inner tube species. The color represents the PL intensity on a linear scale. The values of $n$ and $m$ indicate the chirality (or structure) of the nanotube (Refs. 12-14).

DNA/DWNT solutions with $p \mathrm{H}$ of 2.0 was not easily dispersed in DNA solutions with a vortex mixer, which indicates that the reversibility of the agglomerate is very sensitive to $p \mathrm{H}$.

The mechanism of preferential aggregation of largediameter tubes can be explained by studying their binding interaction with DNA as well as their solubility at low $p \mathrm{H}$. First, we suspected the mechanism to be done to an acidcatalyzed hydrolysis of DNA since it is well known that DNA can be easily damaged by strong acids ( $p \mathrm{H}$ below 2$){ }^{16}$ Therefore, we measured the ratios (the optical absorption intensity at $260 \mathrm{~nm}$ divided by the absorption intensity at 280 $\mathrm{nm})$ for DNA and DNA/DWNT solutions prepared at different $p \mathrm{H}$ since this ratio has been widely utilized as an indicator for DNA purity. ${ }^{17}$ However, we found no distinctive changes in the ratio values, which indicates the absence of $\mathrm{C}-\mathrm{N}$ cleavages even in strong acidic environments. In addition, we evaluated the changes in the solubility of DNA in aqueous solutions when the $p \mathrm{H}$ was decreased to 1.7 , where the clear change from a transparent DNA solution to an opaque milklike DNA solution indicated the reduced solubility of DNA at low $p \mathrm{H}$ due to the strong and extensive protonation of the backbone phosphate groups within the DNA molecule. However, we did not observe agglomerates in either the SWNT solutions containing random and long DNA sequences or the $d(\mathrm{GT})_{15}$-dispersed DWNT solutions, even when the $p \mathrm{H}$ was decreased to 1.7. Based on the aforemen- tioned experimental results, we expect that the stronger van der Waals forces between large-diameter tubes, combined with the decreased solubility of DNA in water at low $p \mathrm{H}$, lead to the preferential agglomeration of large-diameter tubes, which allows the diameter-selective separation of DWNTs to be carried out.

As a result, it was demonstrated that the selected long and random DNA molecules are useful for isolating DWNTs and are only effective for separating DWNTs as a function of their diameter via the controlled solubility of DNA at low $p \mathrm{H}$. We believe that samples containing either large-diameter or small-diameter DWNTs in solutions will pave the way to the simple preparation of individualized specific DWNT configurations.

This work was supported in part by the CLUSTER project (the second stage) of the Ministry of Education, Culture, Sports, Science and Technology of Japan. Y.A.K. acknowledges support from a Grant-in-Aid from the Ministry of Education, Culture, Sports, Science and Technology of Japan (Grant No. 20510096). M.S.D. acknowledges support from NSF Grant No. DMR 07-04197. We also thank CONACYT-Mexico for Grant Nos. 56787 (Laboratory for Nanoscience and Nanotechnology Research-LINAN), 45772 (M.T.), 41464 Inter American Collaboration (M.T.), and 2004-01-013 SALUD-CONACYT (M.T.).

${ }^{1}$ M. S. Arnold, A. A. Green, J. F. Hulvat, S. I. Stupp, and M. C. Hersam, Nat. Nanotechnol. 1, 60 (2006).

${ }^{2}$ A. Nish, J.-Y. Hwang, J. Doig, and A. R. Nicholas, Nat. Nanotechnol. 2, 640 (2007).

${ }^{3}$ M. C. LeMieux, M. Roberts, S. Barman, Y. W. Jin, J. M. Kim, and Z. Bao, Science 321, 101 (2008).

${ }^{4}$ M. Zheng, A. Jagota, E. D. Semke, B. A. Diner, R. S. Mclean, S. R. Lustig, R. E. Richardson, and N. G. Tassi, Nature Mater. 2, 338 (2003).

${ }^{5}$ M. Zheng, A. Jagota, M. S. Strano, A. P. Santos, P. Barone, S. G. Cho, B. A. Diner, M. S. Dresselhaus, R. S. Mclean, G. B. Onoa, G. G. Samsonidze, E. D. Semke, M. Usrey, and D. J. Walls, Science 302, 1545 (2003).

${ }^{6}$ S. G. Chou, H. B. Ribeiro, E. B. Barros, A. P. Santos, D. Nezich, Ge. G. Samsonidze, C. Fantini, M. A. Pimenta, A. Jorio, F. Plentz Filho, M. S. Dresselhaus, G. Dresselhaus, R. Saito, M. Zheng, G. B. Onoa, E. D. Semke, A. K. Swan, M. S. Ünlü, and B. B. Goldberg, Chem. Phys. Lett. 397, 296 (2004).

${ }^{7}$ M. S. Strano, M. Zheng, A. Jagota, G. B. Onoa, D. A. Heller, P. W. Barone, and M. L. Usrey, Nano Lett. 4, 543 (2004).

${ }^{8}$ S. Meng, P. Maragakis, C. Papaloukas, and E. Kaxiras, Nano Lett. 7, 45 (2007).

${ }^{9}$ X. Zhao and J. K. Johnson, J. Am. Chem. Soc. 129, 10438 (2007).

${ }^{10}$ M. Endo, H. Muramatsu, T. Hayashi, Y. A. Kim, M. Terrones, and M. S. Dresselhaus, Nature (London) 433, 476 (2005).

${ }^{11}$ Y. A. Kim, H. Muramatsu, T. Hayashi, M. Endo, M. Terrones, and M. S Dresselhaus, Chem. Phys. Lett. 398, 87 (2004).

${ }^{12}$ M. S. Strano, C. B. Huffman, V. C. Moore, M. J. O'Connell, E. H. Haroz, J. Hubbard, M. Miller, K. Rialon, C. Kittrell, S. Ramesh, R. H. Hauge, and R. E. Smalley, J. Phys. Chem. B 107, 6979 (2003).

${ }^{13}$ M. J. O'Connell, S. M. Bachilo, C. B. Huffman, V. C. Moore, M. S. Strano, E. H. Haroz, K. L. Rialon, P. J. Boul, W. H. Noon, C. Kittrell, J. Ma, R. H. Hauge, R. Bruce Weisman, and R. E. Smalley, Science 297, 593 (2002).

${ }^{14}$ R. Saito, G. Dresselhaus, and M. S. Dresselhaus, Physical Properties of Carbon Nanotubes (Imperial College Press, New York, 1998).

${ }^{15}$ Y. Ohno, S. Iwasaki, Y. Murakami, S. Kishimoto, S. Maruyama, and T. Mizutani, Phys. Rev. B 73, 235427 (2006).

${ }^{16}$ L. Hevesi, E. Wolfson-Davidson, J. B. Nagy, O. B. Nagy, and A. Bruylants, J. Am. Chem. Soc. 94, 4715 (1972).

${ }^{17}$ J. A. Lasel, BioTechniques 18, 62 (1995). 